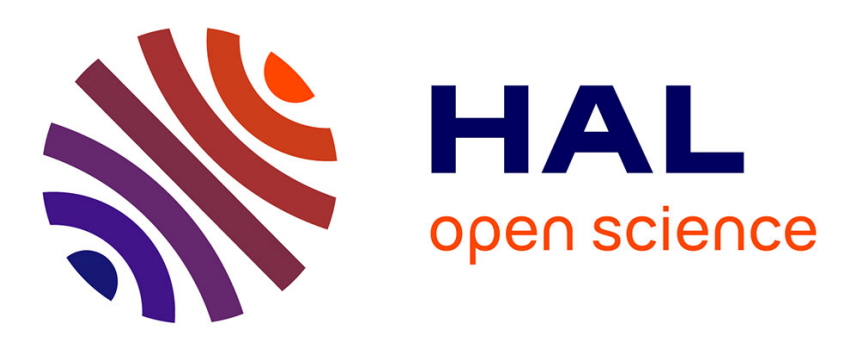

\title{
Kalman filtering with unknown inputs via optimal state estimation of singular systems
}

\author{
Mohamed Darouach, Michel Zasadzinski, André Bassong Onana, Samuel \\ Nowakowski
}

\section{- To cite this version:}

Mohamed Darouach, Michel Zasadzinski, André Bassong Onana, Samuel Nowakowski. Kalman filtering with unknown inputs via optimal state estimation of singular systems. International Journal of Systems Science, 1995, 26 (10), pp.2015-2028. hal-00143941

\section{HAL Id: hal-00143941 https://hal.science/hal-00143941}

Submitted on 28 Apr 2007

HAL is a multi-disciplinary open access archive for the deposit and dissemination of scientific research documents, whether they are published or not. The documents may come from teaching and research institutions in France or abroad, or from public or private research centers.
L'archive ouverte pluridisciplinaire HAL, est destinée au dépôt et à la diffusion de documents scientifiques de niveau recherche, publiés ou non, émanant des établissements d'enseignement et de recherche français ou étrangers, des laboratoires publics ou privés. 


\title{
Kalman filtering with unknown inputs via optimal state estimation of singular systems
}

\author{
M. DAROUACH, M. ZASADZINSKI, A. BASSONG ONANA and S. NOWAKOWSKI \\ CRAN (CNRS UA 821) \\ Université de NANCY I
}

186 rue de Lorraine, 54400 COSNES ET ROMAIN, FRANCE

\begin{abstract}
A new method for designing a Kalman filter for linear discrete-time systems with unkown inputs is presented. The algorithm recently developed for stochastic singular systems is applied to obtain a linear estimation of the state and unkown inputs. The necessary and sufficient conditions for the existence and stability of the filter are derived and proved. An illustrative example is included.
\end{abstract}

\section{Introduction}

In order to estimate the state of stochastic dynamical systems from the Kalman filter, it is generally assumed that all system parameters, noise covariances, and inputs are known. In practice, there exist many situations in which this assumption does not hold. Indeed, the standard Kalman filtering technique may fail in the presence of parametric uncertainties, noise covariance uncertainties, or unmeasurable inputs.

Since decades, many authors have investigated the effect of perturbations on standard estimation procedures. In the strict point of view of the state estimation in presence of unknown inputs, a large number of investigators have focused their attention on building full-order or reduced-order observers for determinist systems (Hou and Müller 1992). One approach developed consists of modeling the unknown inputs by the response of a suitably chosen dynamical system . This method, however, increases the dimension of the observer considerably. More interesting is the approach developed by Wang et al. (1975), Yang and Wilde (1988), Guan and Saif (1991) and Hou and Müller (1992), since the knowledge of no a priori information about the nature of the unknown inputs is required. In the stochastic case, unfortunately, a few results have been obtained for the estimation of state and unknown inputs. Most of these results are either suboptimal (Singer 1970, Gholson and Moose 1977, Chang and Tabaczynski 1984), instable (Myers and Tappley 1976, Kirlin and Maghaddamjoo 1986), or require the generation of the unknown inputs (Maghaddamjoo and Kirlin 1989).

In this paper, we present a new approach for the Kalman filtering with unknown inputs. The problem is formulated as the state estimation of stochastic singular systems with no assumption about the knowledge of the unknown inputs.

The paper is organized as follows. In section 2, a general problem of Kalman filtering with unknown inputs is formulated as a problem of state estimation of singular systems. In section 3 , we give the main results on the singular systems state estimation, as well as stability conditions. In section 4, these results are applied to the Kalman filtering with unknown inputs, and the most general 
conditions, assumed for the existence of the unknown inputs observers, are shown to be necessary and sufficient for the stability of the obtained filter. In section 5, an illustrative example is presented.

\section{Problem statement and basic assumptions}

Consider the following linear time-invariant stochastic discrete system

$$
\begin{aligned}
& \mathrm{x}_{\mathrm{k}+1}=\mathrm{Ax}_{\mathrm{k}}+\mathrm{B} \mathrm{u}_{\mathrm{k}}+\mathrm{Fd}_{\mathrm{k}}+\mathrm{w}_{\mathrm{k}} \\
& \mathrm{y}_{\mathrm{k}}=\mathrm{Hx}_{\mathrm{k}}+\mathrm{v}_{\mathrm{k}}
\end{aligned}
$$

where the state vector $\mathrm{x}_{\mathrm{k}} \in \mathfrak{R}^{\mathrm{n}}$, the control input vector $\mathrm{u}_{\mathrm{k}} \in \mathfrak{R}^{\mathrm{m}}$, the unmeasurable disturbance or unknown input vector $\mathrm{d}_{\mathrm{k}} \in \mathfrak{R}^{\mathrm{q}}$ and the output vector $\mathrm{y}_{\mathrm{k}} \in \mathfrak{R}^{\mathrm{p}}$. Matrices $\mathrm{A}, \mathrm{B}, \mathrm{F}$ and $\mathrm{H}$ have appropriate dimension. $\mathrm{v}_{\mathrm{k}}$ and $\mathrm{w}_{\mathrm{k}}$ are zero mean white sequences uncorrelated with each other and with the initial state of the system. Their covariances are given as

$$
\mathrm{E}\left(\mathrm{w}_{\mathrm{k}} \mathrm{w}_{\mathrm{j}}^{\mathrm{T}}\right)=\mathrm{W} \delta_{\mathrm{kj}}, \mathrm{E}\left(\mathrm{v}_{\mathrm{k}} \mathrm{v}_{\mathrm{j}}^{\mathrm{T}}\right)=\mathrm{V} \delta_{\mathrm{kj}}, \mathrm{W}>0 \text { and } \mathrm{V}>0
$$

where $\delta_{\mathrm{kj}}$ is the Kronecker delta.

The problem is to estimate the state vector $x_{k}$ and the unknown input $d_{k}$ from the Kalman filtering, assuming no knowledge about the vector $\mathrm{d}_{\mathrm{k}}$. This can be made under the following assumptions

$$
\begin{aligned}
& \text { A1 }-\operatorname{rank}(H)=p \\
& \text { A2 }-\operatorname{rank}(F)=q \\
& \text { A3 }-\mathrm{q} \leq \mathrm{p} \\
& \text { A4 }-\operatorname{rank}(H F)=q
\end{aligned}
$$

These assumptions are generally made in unknown input observers. Assumption 2 ( $\mathrm{F}$ has full column rank q) is not a restriction and can be always met by redefining the unknown input vector as follows. If rank $(F)=q_{1}<q$, then $F$ can be expressed as $F=F_{1} N$ where $F_{1}$ has full column rank $q_{1}$ and $\mathrm{d}_{1}=\mathrm{Nd}$ is the new $\left(\mathrm{q}_{1} \times 1\right)$ unknown vector.

System (1)-(2) can be expressed in the following singular system form

$$
\begin{aligned}
& \mathcal{E} X_{\mathrm{k}+1}=\mathcal{A} X_{\mathrm{k}}+\mathcal{B} \mathrm{u}_{\mathrm{k}}+\mathrm{w}_{\mathrm{k}} \\
& \mathrm{y}_{\mathrm{k}}=\mathcal{H} X_{\mathrm{k}}+\mathrm{v}_{\mathrm{k}}
\end{aligned}
$$

where $X_{\mathrm{k}}=\left[\begin{array}{l}\mathrm{x}_{\mathrm{k}} \\ \mathrm{d}_{\mathrm{k}-1}\end{array}\right], \mathcal{E}=\left[\begin{array}{ll}\mathrm{I} & -\mathrm{F}\end{array}\right], \mathcal{A}=\left[\begin{array}{ll}\mathrm{A} & 0\end{array}\right], \mathcal{B}=\mathrm{B}$ and $\mathcal{H}=\left[\begin{array}{ll}\mathrm{H} & 0\end{array}\right]$.

The problem of state and unknown input estimation is reduced to one of the semi-state estimation of the singular system (3)-(4 ). From the above assumptions we have

$$
\operatorname{rank}\left(\left[\begin{array}{c}
\mathcal{E} \\
\mathcal{H}
\end{array}\right]\right)=\mathrm{n}+\mathrm{q}
$$


since

$$
\begin{aligned}
\operatorname{rank}\left(\left[\begin{array}{c}
\mathcal{E} \\
\mathcal{H}
\end{array}\right]\right) & =\operatorname{rank}\left(\left[\begin{array}{cc}
\mathrm{I} & -\mathrm{F} \\
\mathrm{H} & 0
\end{array}\right]\right) \\
& =\operatorname{rank}\left(\left[\begin{array}{cc}
\mathrm{I} & 0 \\
-\mathrm{H} & \mathrm{I}
\end{array}\right]\left[\begin{array}{cc}
\mathrm{I} & -\mathrm{F} \\
\mathrm{H} & 0
\end{array}\right]\right) \\
& =\operatorname{rank}\left(\left[\begin{array}{cc}
\mathrm{I} & -\mathrm{F} \\
0 & \mathrm{HF}
\end{array}\right]\right) \\
& =\mathrm{n}+\operatorname{rank}(\mathrm{HF})
\end{aligned}
$$

Now consider the following stochastic singular system

$$
\begin{aligned}
& \mathrm{Ex}_{\mathrm{k}+1}=\mathrm{Ax}_{\mathrm{k}}+\mathrm{Bu}_{\mathrm{k}}+\mathrm{w}_{\mathrm{k}} \\
& \mathrm{y}_{\mathrm{k}}=\mathrm{H}_{\mathrm{k}}+\mathrm{v}_{\mathrm{k}}
\end{aligned}
$$

where the state vector $x_{k} \in \Re^{n}$, the control input vector $u_{k} \in \Re^{r}$, the output vector $y_{k} \in \Re^{m}$ and matrix $\mathrm{E} \in \mathfrak{R}^{\mathrm{pxn}}$. Matrices $\mathrm{A}, \mathrm{B}, \mathrm{F}$ and $\mathrm{H}$ are of appropriate dimensions. $\mathrm{v}_{\mathrm{k}}$ and $\mathrm{w}_{\mathrm{k}}$ are zero mean white noises with

$$
\mathrm{E}\left(\mathrm{w}_{\mathrm{k}} \mathrm{w}_{\mathrm{j}}^{\mathrm{T}}\right)=\mathrm{W} \delta_{\mathrm{kj}}, \mathrm{E}\left(\mathrm{v}_{\mathrm{k}} \mathrm{v}_{\mathrm{j}}^{\mathrm{T}}\right)=\mathrm{V} \delta_{\mathrm{kj}}, \mathrm{W}>0 \text { and } \mathrm{V}>0
$$

Under the above assumptions and $\left.\operatorname{rank}\left(\begin{array}{l}E \\ H\end{array}\right]\right)=n$, the optimal state estimator for the singular system (6)-(7), is given by the following recursion (Zasadzinski et al. 1991, Nikoukhah et al. 1992, Darouach et al. 1993)

$$
\hat{\mathrm{x}}_{\mathrm{k}+1 / \mathrm{k}+1}=\mathrm{P}_{\mathrm{k}+1 / \mathrm{k}+1}\left(\mathrm{E}^{\mathrm{T}}\left(\mathrm{W}+\mathrm{A} \mathrm{P}_{\mathrm{k} / \mathrm{k}} \mathrm{A}^{\mathrm{T}}\right)^{-1}\left(\mathrm{~A} \hat{\mathrm{x}}_{\mathrm{k} / \mathrm{k}}+\mathrm{B} \mathrm{u}_{\mathrm{k}}\right)+\mathrm{H}^{\mathrm{T}} \mathrm{V}^{-1} \mathrm{y}_{\mathrm{k}+1}\right)
$$

where $\hat{x}_{\mathrm{k} / \mathrm{k}}$ represents the estimate of $\mathrm{x}_{\mathrm{k}}$ based on the measurements up to time instant $\mathrm{k}$ and

$$
\mathrm{P}_{\mathrm{k} / \mathrm{k}}=\mathrm{E}\left(\hat{\mathrm{x}}_{\mathrm{k} / \mathrm{k}}-\mathrm{x}_{\mathrm{k}}\right)\left(\hat{\mathrm{x}}_{\mathrm{k} / \mathrm{k}}-\mathrm{x}_{\mathrm{k}}\right)^{\mathrm{T}}
$$

is the estimation error covariance matrix, subject to the following Generalized Riccati Difference Equation (GRDE)

$$
\mathrm{P}_{\mathrm{k}+1 / \mathrm{k}+1}=\left(\mathrm{E}^{\mathrm{T}}\left(\mathrm{W}+\mathrm{AP}_{\mathrm{k} / \mathrm{k}} \mathrm{A}^{\mathrm{T}}\right)^{-1} \mathrm{E}+\mathrm{H}^{\mathrm{T}} \mathrm{V}^{-1} \mathrm{H}\right)^{-1}
$$

\section{Main results}

To analyze the stability of the filter (8)-(10) we use the singular value decomposition of the matrix $\left[\begin{array}{c}E \\ H\end{array}\right]$. This yields an equivalent standard Kalman filter and its associated Riccati difference equation for which the convergence and stability conditions are well established (De Souza et al. 1986). In order to do this, we first need a number of preliminary results and definitions.

Since $\left.\operatorname{rank}\left(\begin{array}{l}E \\ H\end{array}\right]\right)=n$, there exist orthogonal matrices $U \in \mathfrak{R}^{(m+p)(m+p)}$ and $T \in \mathfrak{R}^{\text {nxm }}$ such that 


$$
\left[\begin{array}{l}
\mathrm{E} \\
\mathrm{H}
\end{array}\right]=\mathrm{U}\left[\begin{array}{l}
\Sigma \\
0
\end{array}\right] \mathrm{T}^{\mathrm{T}}
$$

where $\Sigma=\operatorname{diag}\left(\sigma_{1}, \sigma_{2}, \ldots, \sigma_{n}\right)$. The $\sigma_{i}$ 's are the non zero singular values of $\left[\begin{array}{l}E \\ H\end{array}\right]$. We define the following matrices

$$
\begin{aligned}
& {\left[\begin{array}{l}
\mathrm{A}_{1} \\
\mathrm{~A}_{2}
\end{array}\right]=\mathrm{U}^{\mathrm{T}}\left[\begin{array}{l}
\mathrm{A} \\
0
\end{array}\right],\left[\begin{array}{l}
\mathrm{M}_{1} \\
\mathrm{M}_{2}
\end{array}\right]=\mathrm{U}^{\mathrm{T}}\left[\begin{array}{l}
0 \\
\mathrm{I}
\end{array}\right],\left[\begin{array}{l}
\mathrm{B}_{1} \\
\mathrm{~B}_{2}
\end{array}\right]=\mathrm{U}^{\mathrm{T}}\left[\begin{array}{l}
\mathrm{B} \\
0
\end{array}\right],} \\
& \mathrm{G}=\mathrm{T} \Sigma^{-1}, \mathrm{~F}=\mathrm{G} \mathrm{A}_{1}, \mathrm{C}=\mathrm{A}_{2}, \mathrm{M}=\mathrm{GM}_{1}, \mathrm{D}=\mathrm{GB}_{1}, \\
& {\left[\begin{array}{cc}
\mathrm{Q} & \mathrm{S} \\
\mathrm{S}^{\mathrm{T}} & \mathrm{R}
\end{array}\right]=\left[\begin{array}{ll}
\mathrm{G} & 0 \\
0 & \mathrm{I}
\end{array}\right] \mathrm{U}^{\mathrm{T}}\left[\begin{array}{cc}
\mathrm{W} & 0 \\
0 & \mathrm{~V}
\end{array}\right] \mathrm{U}\left[\begin{array}{cc}
\mathrm{G}^{\mathrm{T}} & 0 \\
0 & \mathrm{I}
\end{array}\right], \mathrm{Q}^{\mathrm{S}}=\mathrm{Q}-\mathrm{S} \mathrm{R}^{-1} \mathrm{~S}^{\mathrm{T}} \text { and } \mathrm{F}^{\mathrm{S}}=\mathrm{F}-\mathrm{S} \mathrm{R}^{-1} \mathrm{C}}
\end{aligned}
$$

where matrices $A_{1}, B_{1}$ and $M_{1}$ have $n$ rows. We can now derive the following results.

\section{Theorem 3.1}

Assume that $\left.\operatorname{rank}\left[\begin{array}{l}E \\ H\end{array}\right]\right)=n$. The optimal filter of system (6)-(7) is given by the following recursions

$$
\hat{\mathrm{x}}_{\mathrm{k}+1 / \mathrm{k}+1}=\mathrm{F}^{\mathrm{s}} \hat{\mathrm{x}}_{\mathrm{k} / \mathrm{k}}+\mathrm{M} \mathrm{y}_{\mathrm{k}+1}+\mathrm{D} \mathrm{u}_{\mathrm{k}}-\mathrm{K}_{\mathrm{k}}^{\mathrm{s}}\left(\mathrm{M}_{2} \mathrm{y}_{\mathrm{k}+1}+\mathrm{B}_{2} \mathrm{u}_{\mathrm{k}}+\mathrm{C} \hat{\mathrm{x}}_{\mathrm{k} / \mathrm{k}}\right)
$$

where

$$
\mathrm{K}_{\mathrm{k}}^{\mathrm{s}}=\mathrm{F}^{\mathrm{S}} \mathrm{P}_{\mathrm{k} / \mathrm{k}} \mathrm{C}^{\mathrm{T}}\left(\mathrm{R}+\mathrm{CP}_{\mathrm{k} / \mathrm{k}} \mathrm{C}^{\mathrm{T}}\right)^{-1}
$$

and $\mathrm{P}_{\mathrm{k} / \mathrm{k}}$ satisfies the Riccati difference equation

$$
\mathrm{P}_{\mathrm{k}+1 / \mathrm{k}+1}=\mathrm{Q}^{\mathrm{s}}+\mathrm{F}^{\mathrm{s}} \mathrm{P}_{\mathrm{k} / \mathrm{k}} \mathrm{F}^{\mathrm{sT}}-\mathrm{F}^{\mathrm{S}} \mathrm{P}_{\mathrm{k} / \mathrm{k}} \mathrm{C}^{\mathrm{T}}\left(\mathrm{R}+\mathrm{CP}_{\mathrm{k} / \mathrm{k}} \mathrm{C}^{\mathrm{T}}\right)^{-1} \mathrm{CP}_{\mathrm{k} / \mathrm{k}} \mathrm{F}^{\mathrm{sT}}
$$

\section{Proof}

The optimal state estimator for system (6)-(7), given by (8)-(9) can be written as $\hat{\mathrm{x}}_{\mathrm{k}+1 / \mathrm{k}+1}=\mathrm{P}_{\mathrm{k}+1 / \mathrm{k}+1}\left[\begin{array}{ll}\mathrm{E}^{\mathrm{T}} & \mathrm{H}^{\mathrm{T}}\end{array}\right]\left(\left[\begin{array}{cc}\mathrm{W} & 0 \\ 0 & \mathrm{~V}\end{array}\right]+\left[\begin{array}{c}\mathrm{A} \\ 0\end{array}\right] \mathrm{P}_{\mathrm{k} / \mathrm{k}}\left[\begin{array}{ll}\mathrm{A}^{\mathrm{T}} & 0\end{array}\right]\right)^{-1}\left(\left[\begin{array}{c}\mathrm{A} \\ 0\end{array}\right] \hat{\mathrm{x}}_{\mathrm{k} / \mathrm{k}}+\left[\begin{array}{l}0 \\ \mathrm{I}\end{array}\right] \mathrm{y}_{\mathrm{k}+1}+\left[\begin{array}{c}\mathrm{B} \\ 0\end{array}\right] \mathrm{u}_{\mathrm{k}}\right)$

and

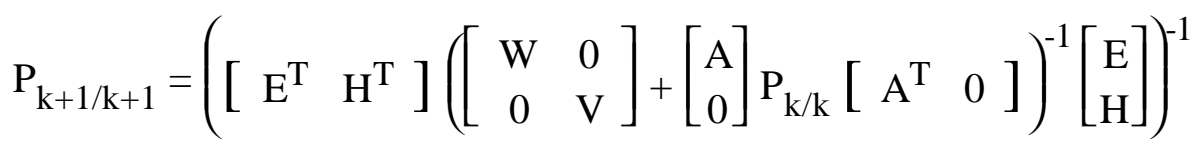

Using the above singular value decomposition and the above matrices we obtain

$$
\mathrm{P}_{\mathrm{k}+1 / \mathrm{k}+1}=\left(\left[\begin{array}{ll}
\mathrm{I} & 0
\end{array}\right]\left[\begin{array}{cc}
\mathrm{Q}+\mathrm{FP}_{\mathrm{k} / \mathrm{k}} \mathrm{F}^{\mathrm{T}} & \mathrm{S}+\mathrm{FP}_{\mathrm{k} / \mathrm{k}} \mathrm{C}^{\mathrm{T}} \\
\mathrm{S}^{\mathrm{T}}+\mathrm{CP}_{\mathrm{k} / \mathrm{k}} \mathrm{F}^{\mathrm{T}} & \mathrm{R}+\mathrm{CP}_{\mathrm{k} / \mathrm{k}} \mathrm{C}^{\mathrm{T}}
\end{array}\right]^{-1}\left[\begin{array}{l}
\mathrm{I} \\
0
\end{array}\right]\right)^{-1}
$$

and 


$$
\hat{x}_{k+1 / k+1}=P_{k+1 / k+1}\left[\begin{array}{ll}
I & 0
\end{array}\right]\left[\begin{array}{cc}
Q+F P_{k / k} F^{T} & S+F P_{k / k} C^{T} \\
S^{T}+C_{k / k} F^{T} & R+C P_{k / k} C^{T}
\end{array}\right]^{-1}\left(\left[\begin{array}{l}
F \\
C
\end{array}\right] \hat{x}_{k / k}+\left[\begin{array}{l}
M \\
M_{2}
\end{array}\right] y_{k+1}+\left[\begin{array}{l}
D \\
B_{2}
\end{array}\right] u_{k}\right)
$$

Using the partitioned matrix inverse, we obtain the state estimate

$$
\hat{\mathrm{x}}_{\mathrm{k}+1 / \mathrm{k}+1}=\mathrm{F} \hat{\mathrm{x}}_{\mathrm{k} / \mathrm{k}}+\mathrm{My}_{\mathrm{k}+1}+\mathrm{Du}_{\mathrm{k}}-\left(\mathrm{S}+\mathrm{FP}_{\mathrm{k} / \mathrm{k}} \mathrm{C}^{\mathrm{T}}\right)\left(\mathrm{R}+\mathrm{CP}_{\mathrm{k} / \mathrm{k}} \mathrm{C}^{\mathrm{T}}\right)^{-1}\left(\mathrm{M}_{2} \mathrm{y}_{\mathrm{k}+1}+\mathrm{B}_{2} \mathrm{u}_{\mathrm{k}}+\mathrm{C} \hat{\mathrm{x}}_{\mathrm{k} / \mathrm{k}}\right)
$$

and the standard Riccati difference equation

$$
\mathrm{P}_{\mathrm{k}+1 / \mathrm{k}+1}=\mathrm{Q}+\mathrm{FP}_{\mathrm{k} / \mathrm{k}} \mathrm{F}^{\mathrm{T}}-\left(\mathrm{S}+\mathrm{FP}_{\mathrm{k} / \mathrm{k}} \mathrm{C}^{\mathrm{T}}\right)\left(\mathrm{R}+\mathrm{CP}_{\mathrm{k} / \mathrm{k}} \mathrm{C}^{\mathrm{T}}\right)^{-1}\left(\mathrm{~S}^{\mathrm{T}}+\mathrm{CP}_{\mathrm{k} / \mathrm{k}} \mathrm{F}^{\mathrm{T}}\right)
$$

Equations (18) and (19) represent the standard Kalman filter and its Riccati difference equation with correlated measurement and model errors (Anderson and Moore 1979, Caines 1988). This case can be treated like the uncorrelated case by introducing matrices $\mathrm{F}^{\mathrm{S}}$ and $\mathrm{Q}^{\mathrm{S}}$ which yields equations (11)-(13).

\section{Remark 3.1}

If $\mathrm{P}_{\mathrm{k} / \mathrm{k}}$ converges as $\mathrm{k} \rightarrow \infty$, the limiting solution $\mathrm{P}$ will satisfy the following Algebraic Riccati Equation (ARE):

$$
\mathrm{P}=\mathrm{Q}^{\mathrm{S}}+\mathrm{F}^{\mathrm{S}} \mathrm{PF}{ }^{\mathrm{sT}}-\mathrm{F}^{\mathrm{S}} \mathrm{PC} \mathrm{C}^{\mathrm{T}}\left(\mathrm{R}+\mathrm{CPC}^{\mathrm{T}}\right)^{-1} \mathrm{CPF}^{\mathrm{sT}}
$$

or equivalently, the Generalized Algebraic Riccati Equation (GARE):

$$
P=\left(E^{T}\left(W+A P A^{T}\right)^{-1} E+H^{T} V^{-1} H\right)^{-1}
$$

We shall be particularly interested by those solutions of the GARE which are real, symmetric and positive semi-definite, and which give a steady-state filter having roots on or inside the unit circle. We therefore introduce the following definition (De Souza et al. 1986).

\section{Definition 3.1}

A real symmetric nonnegative definite solution of the GARE is said to be a stabilizing solution if all roots of the state transition matrix $\left(\mathrm{P}\left(\mathrm{E}^{\mathrm{T}}\left(\mathrm{W}+\mathrm{APA}^{\mathrm{T}}\right)^{-1} \mathrm{~A}\right)\right.$ of the filter $(8)$ and $(10)$ are inside the unit circle.

The necessary and sufficient conditions of the convergence of the GRDE (10) and the stability of (8) can be given from the well established results in the standard systems and theorem 1. 
Theorem 3.2
If $\operatorname{rank}\left(\left[\begin{array}{c}\mathrm{E} \\ \mathrm{H}\end{array}\right]\right)=\mathrm{n}$ and $\mathrm{P}_{0}>0$, then

$$
\operatorname{rank}\left(\left[\begin{array}{c}
\mathrm{zE}-\mathrm{A} \\
\mathrm{H}
\end{array}\right]\right)=\mathrm{n}, \forall \mathrm{z} \in \mathbb{C},|\mathrm{z}| \geq 1
$$

is a necessary and sufficient condition for

$$
\lim _{\mathrm{k} \rightarrow \infty} \mathrm{P}_{\mathrm{k}}=\mathrm{P} \text { (exponentially fast) }
$$

where $\mathrm{P}_{\mathrm{k}}$ is the solution of the GRDE with initial condition $\mathrm{P}_{0}$ and $\mathrm{P}$ is the unique stabilizing solution of the GARE.

\section{Remark 3.2}

If $\mathrm{E}$ is a square matrix, condition (22) corresponds to the detectability of singular systems (Dai 1989).

In order to prove theorem 3.2, we first introduce the following lemma (Hautus 1969, Davis and Vinter 1985).

\section{Lemma 3.1}

The pair (C,F) is detectable if and only if

$$
\left.\operatorname{rank}\left(\begin{array}{c}
\mathrm{zI}-\mathrm{F} \\
\mathrm{C}
\end{array}\right]\right)=\mathrm{n}
$$

for all eigenvalues $\mathrm{z}$ of $\mathrm{F}$ outside the open unit disc.

\section{Proof of theorem 3.2}

From De Souza et al. (1986), subject to $\mathrm{P}_{0}>0$, the RDE (19) has a unique stabilizing solution if and only if $\left(\mathrm{C}, \mathrm{F}^{\mathrm{S}}\right)$ is detectable and $\left(\mathrm{F}^{\mathrm{S}}, \mathrm{N}\right)$ has no unreachable mode on the unit circle $(\mathrm{N}$ is any square root of $\left.\mathrm{Q}^{\mathrm{S}}\right)$. From the above lemma, the detectability of $\left(\mathrm{C}, \mathrm{F}^{\mathrm{S}}\right)$ is equivalent to

$$
\operatorname{rank}\left(\left[\begin{array}{c}
\mathrm{zI}-\mathrm{F}^{\mathrm{s}} \\
\mathrm{C}
\end{array}\right]\right)=\mathrm{n}, \forall \mathrm{z} \in \mathbb{C},|\mathrm{z}| \geq 1
$$

and so

$$
\begin{aligned}
\mathrm{n} & =\operatorname{rank}\left(\left[\begin{array}{cc}
\mathrm{I} & -\mathrm{SR}^{-1} \\
0 & \mathrm{I}
\end{array}\right]\left[\begin{array}{c}
\mathrm{zI}-\mathrm{F}^{\mathrm{s}} \\
\mathrm{C}
\end{array}\right]\right) \\
& \left.=\operatorname{rank}\left(\begin{array}{c}
\mathrm{zI}-\mathrm{F} \\
\mathrm{C}
\end{array}\right]\right)
\end{aligned}
$$

From the definition of $\mathrm{F}$ and $\mathrm{C}$, this is also equivalent to 


$$
\begin{aligned}
\mathrm{n}=\operatorname{rank}\left(\left[\begin{array}{c}
\mathrm{zI}-\mathrm{GA}_{1} \\
\mathrm{~A}_{2}
\end{array}\right]\right) & =\operatorname{rank}\left(\left[\begin{array}{c}
\mathrm{z} \Sigma \mathrm{T}^{\mathrm{T}} \\
0
\end{array}\right]-\left[\begin{array}{c}
\mathrm{A}_{1} \\
\mathrm{~A}_{2}
\end{array}\right]\right) \\
& =\operatorname{rank}\left(\mathrm{zU}\left[\begin{array}{c}
\Sigma \\
0
\end{array}\right] \mathrm{T}^{\mathrm{T}}-\left[\begin{array}{c}
\mathrm{A} \\
0
\end{array}\right]\right) \\
& =\operatorname{rank}\left(\mathrm{z}\left[\begin{array}{c}
\mathrm{E} \\
\mathrm{H}
\end{array}\right]-\left[\begin{array}{c}
\mathrm{A} \\
0
\end{array}\right]\right) \\
& =\operatorname{rank}\left(\left[\begin{array}{c}
\mathrm{zE}-\mathrm{A} \\
\mathrm{H}
\end{array}\right]\right), \forall \mathrm{z} \in \mathbb{C},|\mathrm{z}| \geq 1 .
\end{aligned}
$$

Now, let $\lambda$ be an unreachable mode of $\left(\mathrm{F}^{\mathrm{S}}, \mathrm{N}\right)$, then there exists a row vector $\mathrm{q} \neq 0$ and a scalar $\lambda$ (possibly complex) such that

$$
\left\{\begin{array}{l}
\mathrm{qF}^{\mathrm{s}}=\lambda \mathrm{q} \\
\mathrm{qN}=0
\end{array}\right.
$$

This can be written

$$
\left\{\begin{array}{l}
\mathrm{q}\left(\mathrm{GA}_{1}-\mathrm{SR}^{-1} \mathrm{~A}_{2}\right)=\lambda \mathrm{q} \\
\mathrm{q}\left(\mathrm{Q}-\mathrm{SR}^{-1} \mathrm{~S}^{\mathrm{T}}\right)=0
\end{array}\right.
$$

or equivalently

$$
\left\{\begin{array}{l}
\mathrm{q}\left(\mathrm{G}-\mathrm{SR}^{-1}\right) \mathrm{U}^{\mathrm{T}}\left[\begin{array}{l}
\mathrm{A} \\
0
\end{array}\right]=\lambda \mathrm{q} \\
\mathrm{q}\left(\mathrm{G}-\mathrm{SR}^{-1}\right) \mathrm{U}^{\mathrm{T}}\left[\begin{array}{cc}
\mathrm{W} & 0 \\
0 & \mathrm{~V}
\end{array}\right]=0
\end{array}\right.
$$

Define $\mathrm{v}=\mathrm{q}\left(\mathrm{G}-\mathrm{SR}^{-1}\right) \mathrm{U}^{\mathrm{T}}$. Multiplying $\mathrm{v}$ by $\mathrm{U}\left[\begin{array}{c}\mathrm{G}^{-1} \\ 0\end{array}\right]$ gives

$$
\mathrm{q}=\mathrm{v}\left[\begin{array}{l}
\mathrm{E} \\
\mathrm{H}
\end{array}\right]
$$

Equations (25) becomes

$$
\left\{\begin{array}{l}
\mathrm{v}\left[\begin{array}{c}
\mathrm{A} \\
0
\end{array}\right]=\lambda \mathrm{v}\left[\begin{array}{c}
\mathrm{E} \\
\mathrm{H}
\end{array}\right] \\
\mathrm{v}\left[\begin{array}{cc}
\mathrm{W} & 0 \\
0 & \mathrm{~V}
\end{array}\right]=0
\end{array}\right.
$$

If matrices $\mathrm{V}$ and $\mathrm{W}$ are positive definite, then $\mathrm{v}=0$ and thus, there does not exist unreachable mode of $\left(\mathrm{F}^{\mathrm{S}}, \mathrm{N}\right)$, in particular on the unit circle.

We are now ready to design a Kalman filter with unknown input. 


\section{Application to Kalman filtering with unknown inputs}

In this section, we show how to handle the generalized Kalman filter for the standard system (1)(2) with unknown inputs. Consider the discrete system with unknown input $d_{k}$ described by (1)-(2). We assume that conditions A1-A4 are satisfied. Then, we can apply the above results to estimate the state $x_{k}$ and the unknown input $d_{k}$.

In section 2 we have introduced a new state vector $x_{k}=\left[\begin{array}{c}x_{k} \\ d_{k-1}\end{array}\right]$. We now define its estimate based on the measurement up to $\mathrm{k}$ by $\hat{X}_{\mathrm{k} / \mathrm{k}}=\left[\begin{array}{c}\hat{\mathrm{x}}_{\mathrm{k} / \mathrm{k}} \\ \hat{\mathrm{d}}_{\mathrm{k}-1 / \mathrm{k}}\end{array}\right]$ and the estimation error covariance matrix by

$$
\mathcal{P}_{\mathrm{k} / \mathrm{k}}=\mathrm{E}\left(\hat{X}_{\mathrm{k} / \mathrm{k}}-x_{\mathrm{k}}\right)\left(\hat{x}_{\mathrm{k} / \mathrm{k}}-x_{\mathrm{k}}\right)^{\mathrm{T}}
$$

which is partitioned as follows

$$
\mathcal{P}_{\mathrm{k} / \mathrm{k}}=\left[\begin{array}{ll}
\mathrm{P}_{\mathrm{k} / \mathrm{k}}^{\mathrm{x}} & \mathrm{P}_{\mathrm{k} / \mathrm{k}}^{\mathrm{xd}} \\
\mathrm{P}_{\mathrm{k} / \mathrm{k}}^{\mathrm{dx}} & \mathrm{P}_{\mathrm{k}-1 / \mathrm{k}}^{\mathrm{d}}
\end{array}\right]
$$

where

$$
\mathrm{P}_{\mathrm{k} / \mathrm{k}}^{\mathrm{x}}=\mathrm{E}\left(\hat{\mathrm{x}}_{\mathrm{k} / \mathrm{k}}-\mathrm{x}_{\mathrm{k}}\right)\left(\hat{\mathrm{x}}_{\mathrm{k} / \mathrm{k}}-\mathrm{x}_{\mathrm{k}}\right)^{\mathrm{T}}
$$

is the state estimation error covariance matrix,

$$
\mathrm{P}_{\mathrm{k}-1 / \mathrm{k}}^{\mathrm{d}}=\mathrm{E}\left(\hat{\mathrm{d}}_{\mathrm{k}-1 / \mathrm{k}}-\mathrm{d}_{\mathrm{k}-1}\right)\left(\hat{\mathrm{d}}_{\mathrm{k}-1 / \mathrm{k}}-\mathrm{d}_{\mathrm{k}-1}\right)^{\mathrm{T}}
$$

is the unknown input estimation error covariance matrix,

$$
\mathrm{P}_{\mathrm{k} / \mathrm{k}}^{\mathrm{xd}}=\mathrm{E}\left(\hat{\mathrm{x}}_{\mathrm{k} / \mathrm{k}}-\mathrm{x}_{\mathrm{k}}\right)\left(\hat{\mathrm{d}}_{\mathrm{k}-1 / \mathrm{k}}-\mathrm{d}_{\mathrm{k}-1}\right)^{\mathrm{T}}
$$

is the cross state and unknown input estimation errors covariance matrix with $\mathrm{P}_{\mathrm{k} / \mathrm{k}}^{\mathrm{dx}}=\left(\mathrm{P}_{\mathrm{k} / \mathrm{k}}^{\mathrm{xd}}\right)^{\mathrm{T}}$.

From the generalized Kalman filter ((8) and (10)) and the definition of $\mathcal{E}, \mathrm{A}, \mathcal{B}$, and $\mathcal{H}$, we have

$$
\hat{X}_{\mathrm{k}+1 / \mathrm{k}+1}=\mathcal{P}_{\mathrm{k}+1 / \mathrm{k}+1}\left[\begin{array}{c}
\mathrm{I} \\
-\mathrm{F}^{\mathrm{T}}
\end{array}\right] \overline{\mathrm{P}}_{\mathrm{k} / \mathrm{k}}^{-1} \overline{\mathrm{x}}_{\mathrm{k} / \mathrm{k}}+\mathcal{P}_{\mathrm{k}+1 / \mathrm{k}+1}\left[\begin{array}{c}
\mathrm{H}^{\mathrm{T}} \mathrm{V}^{-1} \\
0
\end{array}\right] \mathrm{y}_{\mathrm{k}+1}
$$

where

$$
\begin{aligned}
& \mathcal{P}_{\mathrm{k}+1 / \mathrm{k}+1}^{-1}=\left[\begin{array}{cc}
\overline{\mathrm{P}}_{\mathrm{k} / \mathrm{k}}^{-1}+\mathrm{H}^{\mathrm{T}} \mathrm{V}^{-1} \mathrm{H} & -\overline{\mathrm{P}}_{\mathrm{k} / \mathrm{k}}^{-1} \mathrm{~F} \\
-\mathrm{F}^{\mathrm{T}} \mathrm{P}_{\mathrm{k} / \mathrm{k}}^{-1} & \mathrm{~F}^{\mathrm{T}} \overline{\mathrm{P}}_{\mathrm{k} / \mathrm{k}}^{-1} \mathrm{~F}
\end{array}\right] \\
& \overline{\mathrm{x}}_{\mathrm{k} / \mathrm{k}}=\mathrm{A}_{\mathrm{k} / \mathrm{k}}+\mathrm{B} \mathrm{u} \mathrm{u}_{\mathrm{k}} \\
& \overline{\mathrm{P}}_{\mathrm{k} / \mathrm{k}}=\mathrm{A} \mathrm{P}_{\mathrm{k} / \mathrm{k}}^{\mathrm{x}} \mathrm{A}^{\mathrm{T}}+\mathrm{W}
\end{aligned}
$$

Equation (28) yields 


$$
\begin{aligned}
& \hat{\mathrm{x}}_{\mathrm{k}+1 / \mathrm{k}+1}=\left(\mathrm{P}_{\mathrm{k}+1 / \mathrm{k}+1}^{\mathrm{x}}-\mathrm{P}_{\mathrm{k}+1 / \mathrm{k}+1}^{\mathrm{xd}} \mathrm{F}^{\mathrm{T}}\right) \overline{\mathrm{P}}_{\mathrm{k} / \mathrm{k}}^{-1} \overline{\mathrm{x}}_{\mathrm{k} / \mathrm{k}}+\mathrm{P}_{\mathrm{k}+1 / \mathrm{k}+1}^{\mathrm{x}} \mathrm{H}^{\mathrm{T}} \mathrm{V}^{-1} \mathrm{y}_{\mathrm{k}+1} \\
& \hat{\mathrm{d}}_{\mathrm{k} / \mathrm{k}+1}=\left(\mathrm{P}_{\mathrm{k}+1 / \mathrm{k}+1}^{\mathrm{dx}}-\mathrm{P}_{\mathrm{k} / \mathrm{k}+1}^{\mathrm{d}} \mathrm{F}^{\mathrm{T}}\right) \overline{\mathrm{P}}_{\mathrm{k} / \mathrm{k}}^{-1} \overline{\mathrm{x}}_{\mathrm{k} / \mathrm{k}}+\mathrm{P}_{\mathrm{k}+1 / \mathrm{k}+1}^{\mathrm{dx}} \mathrm{H}^{\mathrm{T}} \mathrm{V}^{-1} \mathrm{y}_{\mathrm{k}+1}
\end{aligned}
$$

Now using the inverse of the partitioned matrix (29) of $\mathcal{P}_{\mathrm{k}+1 / \mathrm{k}+1}$, we obtain the following set of difference equations

$$
\begin{aligned}
& \mathrm{P}_{\mathrm{k}+1 / \mathrm{k}+1}^{\mathrm{x}}=\left(\overline{\mathrm{P}}_{\mathrm{k} / \mathrm{k}}^{-1}+\mathrm{H}^{\mathrm{T}} \mathrm{V}^{-1} \mathrm{H}-\overline{\mathrm{P}}_{\mathrm{k} / \mathrm{k}}^{-1} \mathrm{~F}\left(\mathrm{~F}^{\mathrm{T}} \overline{\mathrm{P}}_{\mathrm{k} / \mathrm{k}}^{-1} \mathrm{~F}\right)^{-1} \mathrm{~F}^{\mathrm{T}} \overline{\mathrm{P}}_{\mathrm{k} / \mathrm{k}}^{-1}\right)^{-1} \\
& \mathrm{P}_{\mathrm{k} / \mathrm{k}+1}^{\mathrm{d}}=\left(\mathrm{F}^{\mathrm{T}} \mathrm{H}^{\mathrm{T}}\left(\mathrm{V}+\overline{\mathrm{H}}_{\mathrm{k} / \mathrm{k}} \mathrm{H}^{\mathrm{T}}\right)^{-1} \mathrm{H} \mathrm{F}\right)^{-1} \\
& \mathrm{P}_{\mathrm{k}+1 / \mathrm{k}+1}^{\mathrm{xd}}=\mathrm{P}_{\mathrm{k}+1 / \mathrm{k}+1}^{\mathrm{x}} \overline{\mathrm{P}}_{\mathrm{k} / \mathrm{k}}^{-1} \mathrm{~F}\left(\mathrm{~F}^{\mathrm{T}} \overline{\mathrm{P}}_{\mathrm{k} / \mathrm{k}}^{-1} \mathrm{~F}\right)^{-1} \\
& \mathrm{P}_{\mathrm{k}+1 / \mathrm{k}+1}^{\mathrm{dx}}=\mathrm{P}_{\mathrm{k} / \mathrm{k}+1}^{\mathrm{d}} \mathrm{F}^{\mathrm{T}} \overline{\mathrm{P}}_{\mathrm{k} / \mathrm{k}}^{-1}\left(\overline{\mathrm{P}}_{\mathrm{k} / \mathrm{k}}^{-1}+\mathrm{H}^{\mathrm{T}} \mathrm{V}^{-1} \mathrm{H}\right)^{-1}
\end{aligned}
$$

From these equations we have

$$
\begin{aligned}
& \left(\mathrm{P}_{\mathrm{k}+1 / \mathrm{k}+1}^{\mathrm{x}}-\mathrm{P}_{\mathrm{k}+1 / \mathrm{k}+1}^{\mathrm{xd}} \mathrm{F}^{\mathrm{T}}\right) \overline{\mathrm{P}}_{\mathrm{k} / \mathrm{k}}^{-1}=\mathrm{I}-\mathrm{P}_{\mathrm{k}+1 / \mathrm{k}+1}^{\mathrm{x}} \mathrm{H}^{\mathrm{T}} \mathrm{V}^{-1} \mathrm{H} \\
& \left(\mathrm{P}_{\mathrm{k}+1 / \mathrm{k}+1}^{\mathrm{dx}}-\mathrm{P}_{\mathrm{k} / \mathrm{k}+1}^{\mathrm{d}} \mathrm{F}^{\mathrm{T}}\right) \overline{\mathrm{P}}_{\mathrm{k} / \mathrm{k}}^{-1}=-\mathrm{P}_{\mathrm{k}+1 / \mathrm{k}+1}^{\mathrm{dx}} \mathrm{H}^{\mathrm{T}} \mathrm{V}^{-1} \mathrm{H} \\
& \mathrm{P}_{\mathrm{k}+1 / \mathrm{k}+1}^{\mathrm{x}}=\left(\overline{\mathrm{P}}_{\mathrm{k} / \mathrm{k}}^{-1}+\mathrm{H}^{\mathrm{T}} \mathrm{V}^{-1} \mathrm{H}\right)^{-1}+\mathrm{P}_{\mathrm{k}+1 / \mathrm{k}+1}^{\mathrm{xd}}\left(\mathrm{P}_{\mathrm{k} / \mathrm{k}+1}^{\mathrm{d}}\right)^{-1} \mathrm{P}_{\mathrm{k}+1 / \mathrm{k}+1}^{\mathrm{dx}}
\end{aligned}
$$

Inserting (38) and (39) in (32) and (33), we obtain

$$
\begin{aligned}
& \hat{\mathrm{x}}_{\mathrm{k}+1 / \mathrm{k}+1}=\overline{\mathrm{x}}_{\mathrm{k} / \mathrm{k}}+\mathrm{P}_{\mathrm{k}+1 / \mathrm{k}+1}^{\mathrm{x}} \mathrm{H}^{\mathrm{T}} \mathrm{V}^{-1}\left(\mathrm{y}_{\mathrm{k}+1}-\mathrm{H} \overline{\mathrm{x}}_{\mathrm{k} / \mathrm{k}}\right) \\
& \hat{\mathrm{d}}_{\mathrm{k} / \mathrm{k}+1}=\mathrm{P}_{\mathrm{k}+1 / \mathrm{k}+1}^{\mathrm{dx}} \mathrm{H}^{\mathrm{T}} \mathrm{V}^{-1}\left(\mathrm{y}_{\mathrm{k}+1}-\mathrm{H} \overline{\mathrm{x}}_{\mathrm{k} / \mathrm{k}}\right)
\end{aligned}
$$

Substituting (40) into (41), and after some standard calculations, we find the following theorem.

\section{Theorem 4.1}

Let a discrete-time system be given by (1)-(2). Assume that the conditions A1-A4 are satisfied, then the optimal state and unknown inputs estimates are given by

$$
\begin{aligned}
& \overline{\mathrm{x}}_{\mathrm{k} / \mathrm{k}}=\mathrm{A} \hat{\mathrm{x}}_{\mathrm{k} / \mathrm{k}}+\mathrm{B} \mathrm{u}_{\mathrm{k}} \\
& \hat{\mathrm{x}}_{\mathrm{k}+1 / \mathrm{k}+1}=\overline{\mathrm{x}}_{\mathrm{k} / \mathrm{k}}+\mathrm{F} \hat{\mathrm{d}}_{\mathrm{k} / \mathrm{k}+1}+\mathrm{K}_{\mathrm{k}+1}^{\mathrm{x}}\left(\mathrm{y}_{\mathrm{k}+1}-\mathrm{H}\left(\overline{\mathrm{x}}_{\mathrm{k} / \mathrm{k}}+\mathrm{F} \hat{\mathrm{d}}_{\mathrm{k} / \mathrm{k}+1}\right)\right) \\
& \hat{\mathrm{d}}_{\mathrm{k} / \mathrm{k}+1}=\mathrm{K}_{\mathrm{k}+1}^{\mathrm{d}}\left(\mathrm{y}_{\mathrm{k}+1}-\mathrm{H} \overline{\mathrm{x}}_{\mathrm{k} / \mathrm{k}}\right)
\end{aligned}
$$

with

$$
\begin{aligned}
\mathrm{K}_{\mathrm{k}+1}^{\mathrm{X}} & =\left(\overline{\mathrm{P}}_{\mathrm{k} / \mathrm{k}}^{-1}+\mathrm{H}^{\mathrm{T}} \mathrm{V}^{-1} \mathrm{H}\right)^{-1} \mathrm{H}^{\mathrm{T}} \mathrm{V}^{-1} \\
\mathrm{~K}_{\mathrm{k}+1}^{\mathrm{d}} & =\mathrm{P}_{\mathrm{k}+1 / \mathrm{k}+1}^{\mathrm{dx}} \mathrm{H}^{\mathrm{T}} \mathrm{V}^{-1}
\end{aligned}
$$

where $\overline{\mathrm{P}}_{\mathrm{k} / \mathrm{k}}, \mathrm{P}_{\mathrm{k}+1 / \mathrm{k}+1}^{\mathrm{dx}}$ and $\mathrm{P}_{\mathrm{k}+1 / \mathrm{k}+1}^{\mathrm{x}}$ are given by (31), (35)-(37) and (40). 
The conditions of convergence of the GRDE (29) and stability of the filter are given by the following theorem.

\section{Theorem 4.2}

The GRDE (29) associated with the Kalman filter with unknown inputs has a unique stabilizing solution if and only if the transfer function from $\mathrm{d}$ to $\mathrm{y}, \mathrm{H}(\mathrm{zI}-\mathrm{A})^{-1} \mathrm{~F}$, is left invertible and strictly minimum phase, i.e

$$
\left.\operatorname{rank}\left(\begin{array}{cc}
\mathrm{zI}-\mathrm{A} & -\mathrm{F} \\
\mathrm{H} & 0
\end{array}\right]\right)=\mathrm{n}+\mathrm{q}, \forall \mathrm{z} \in \mathbb{C},|\mathrm{z}| \geq 1
$$

Proof

From theorem 3.2, under assumptions A1-A4, the GRDE (29) has a unique stabilizing solution if and only if

$$
\left.\operatorname{rank}\left(\begin{array}{cc}
\mathrm{zI}-\mathrm{A} & -\mathrm{zF} \\
\mathrm{H} & 0
\end{array}\right]\right)=\mathrm{n}+\mathrm{q}, \forall \mathrm{z} \in \mathbb{C},|\mathrm{z}| \geq 1
$$

which is equivalent to (48).

\section{Example}

To illustrate the design procedure of the Kalman filter with unknown inputs, let us consider the system described by

$$
\begin{aligned}
& \mathrm{x}_{\mathrm{k}+1}=\mathrm{A} \mathrm{x}_{\mathrm{k}}+\mathrm{B} \mathrm{u}_{\mathrm{k}}+\mathrm{Fd}_{\mathrm{k}}+\mathrm{w}_{\mathrm{k}} \\
& \mathrm{y}_{\mathrm{k}}=\mathrm{Hx}_{\mathrm{k}}+\mathrm{v}_{\mathrm{k}}
\end{aligned}
$$

where the state $\mathrm{x} \in \mathfrak{R}^{3}$, the control input $\mathrm{u} \in \mathfrak{R}$, the unknown input $\mathrm{d} \in \mathfrak{R}$ and the measured output $\mathrm{y}$ $\in \mathfrak{R}^{2}$. Matrices $\mathrm{A}, \mathrm{B}, \mathrm{F}$ and $\mathrm{H}$ are given by

$$
\mathrm{A}=\left[\begin{array}{lll}
0 & 0.6 & 0.075 \\
0.75 & 0 & 0 \\
0 & 0.75 & 0.0375
\end{array}\right], \mathrm{B}=\left[\begin{array}{l}
1 \\
1 \\
0
\end{array}\right], \mathrm{F}=\left[\begin{array}{l}
0 \\
1 \\
1
\end{array}\right], \mathrm{H}=\left[\begin{array}{lll}
1 & 1 & 0 \\
0 & 1 & 1
\end{array}\right]
$$

$\mathrm{v}_{\mathrm{k}}$ and $\mathrm{w}_{\mathrm{k}}$ are zero mean gaussian noise processes, uncorrelated with each other and with $\mathrm{x}_{0}$ and $\mathrm{d}_{0}$. The covariance matrices $\mathrm{V}, \mathrm{W}$ are

$$
\mathrm{V}=\left[\begin{array}{cc}
12 & 0 \\
0 & 12
\end{array}\right] \text { and } \mathrm{W}=\left[\begin{array}{lll}
3 & 0 & 0 \\
0 & 6 & 0 \\
0 & 0 & 9
\end{array}\right]
$$

The initial prediction of the state and of the unknown input are assumed to be zero and their statistical informations are 


$$
\mathrm{P}_{0}^{\mathrm{X}}=\left[\begin{array}{ccc}
10 & 0 & 0 \\
0 & 10 & 0 \\
0 & 0 & 10
\end{array}\right]
$$

Assumptions A1-A4 hold, since $\operatorname{rank}(\mathrm{F})=1$ and $\operatorname{rank}(\mathrm{HF})=1$.

The convergence of the GRDE associated with the Kalman filter with unknown input to the stabilizing solution is guaranteed (theorem 4.2), since the transfer matrix

$$
H(z I-A)^{-1} F=\left[\begin{array}{c}
\frac{z^{2}+0.6375 z+0.09}{z^{3}-0.0375 z^{2}-0.45 z-0.0253} \\
\frac{2 z^{2}+0.7125 z-0.3938}{z^{3}-0.0375 z^{2}-0.45 z-0.0253}
\end{array}\right]
$$

is left invertible and strictly minimum phase.

Figures 1 to 3 give a comparison between the actual and estimated states. Figure 4 gives a plot of the actual unknown input $d$ and its estimate. Figure 5 shows the evolution of the error variances of the estimates.

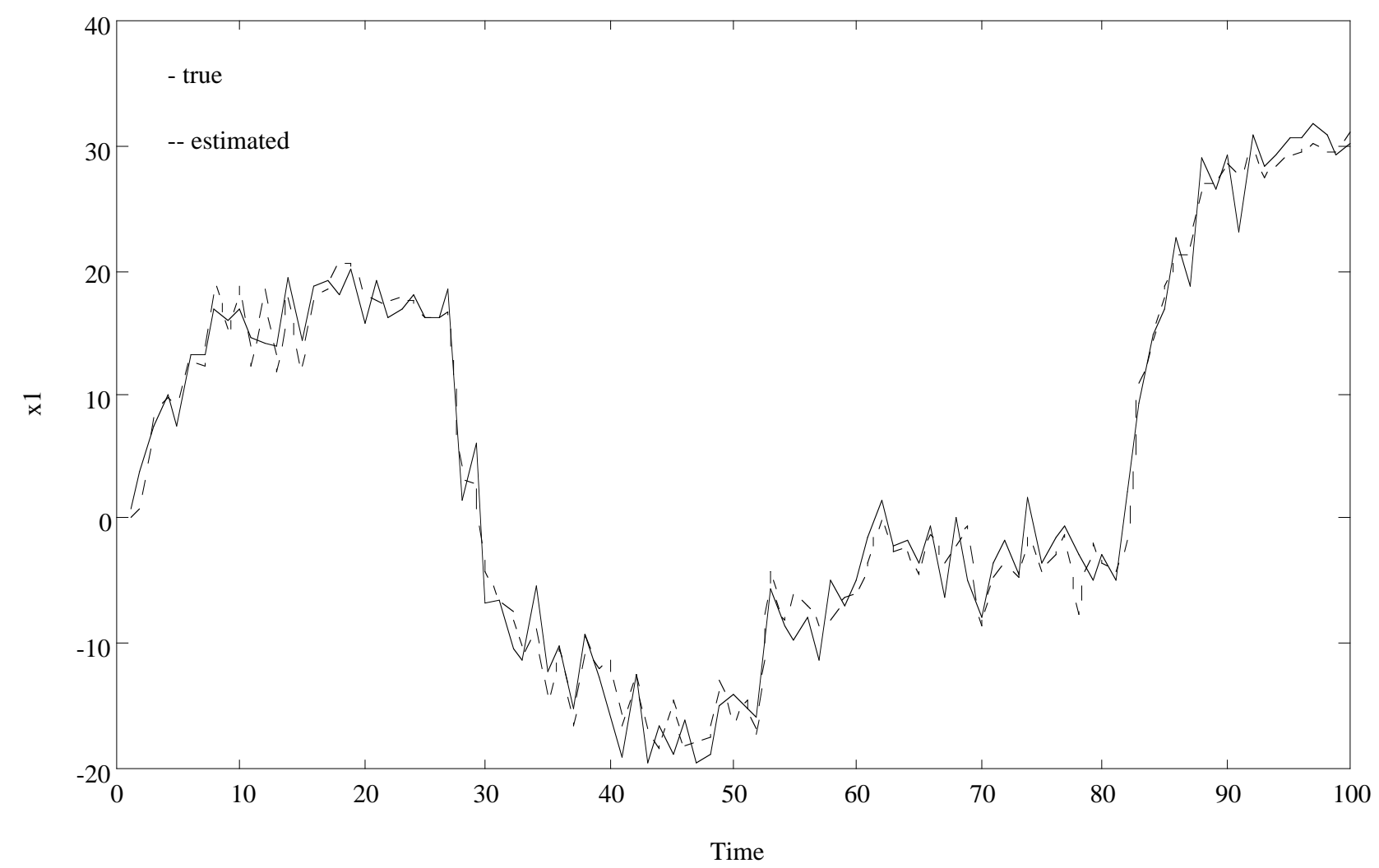

Figure 1. True and estimated state $\mathrm{x}_{1}$ 


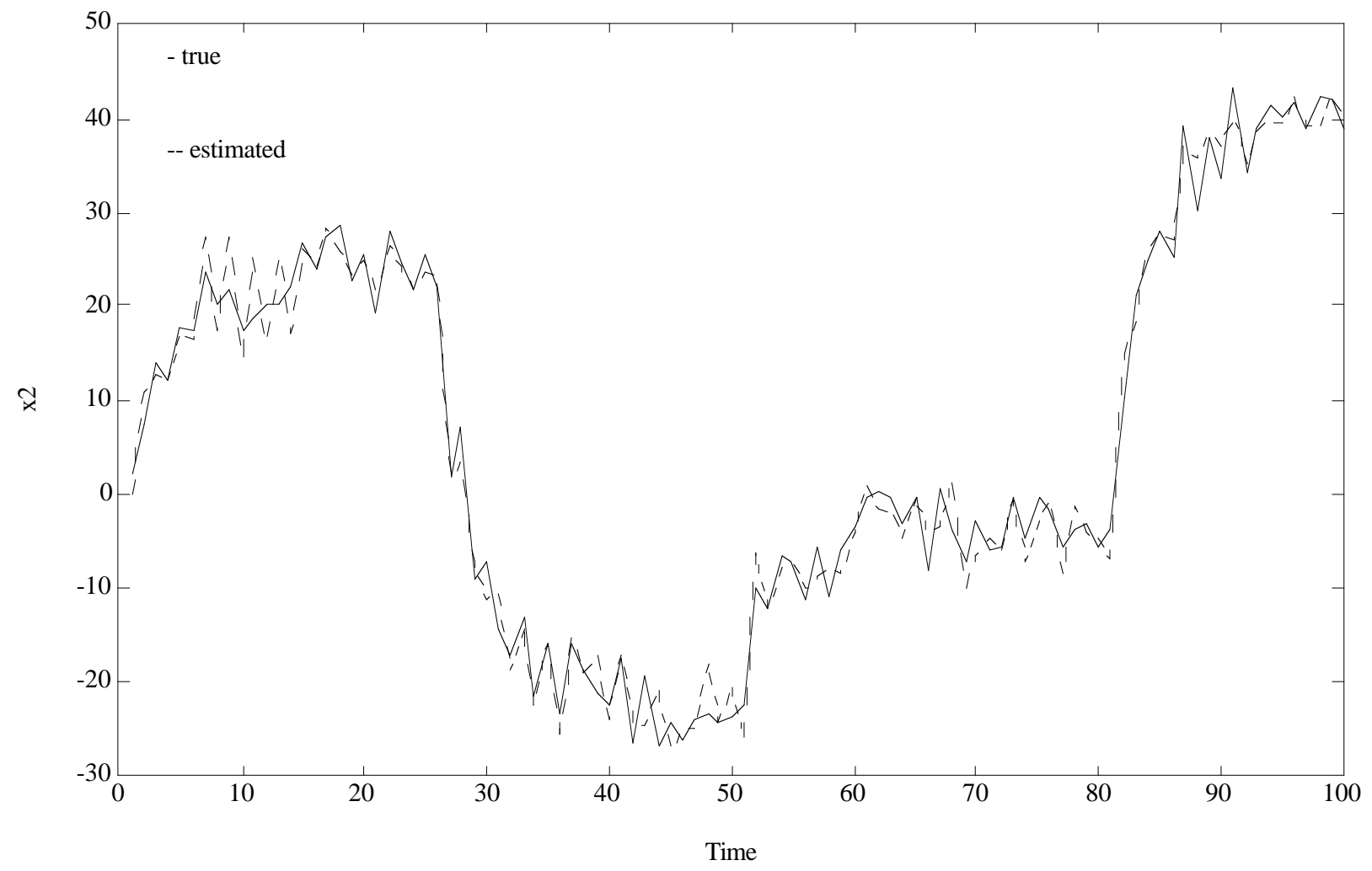

Figure 2. True and estimated state $\mathrm{x}_{2}$

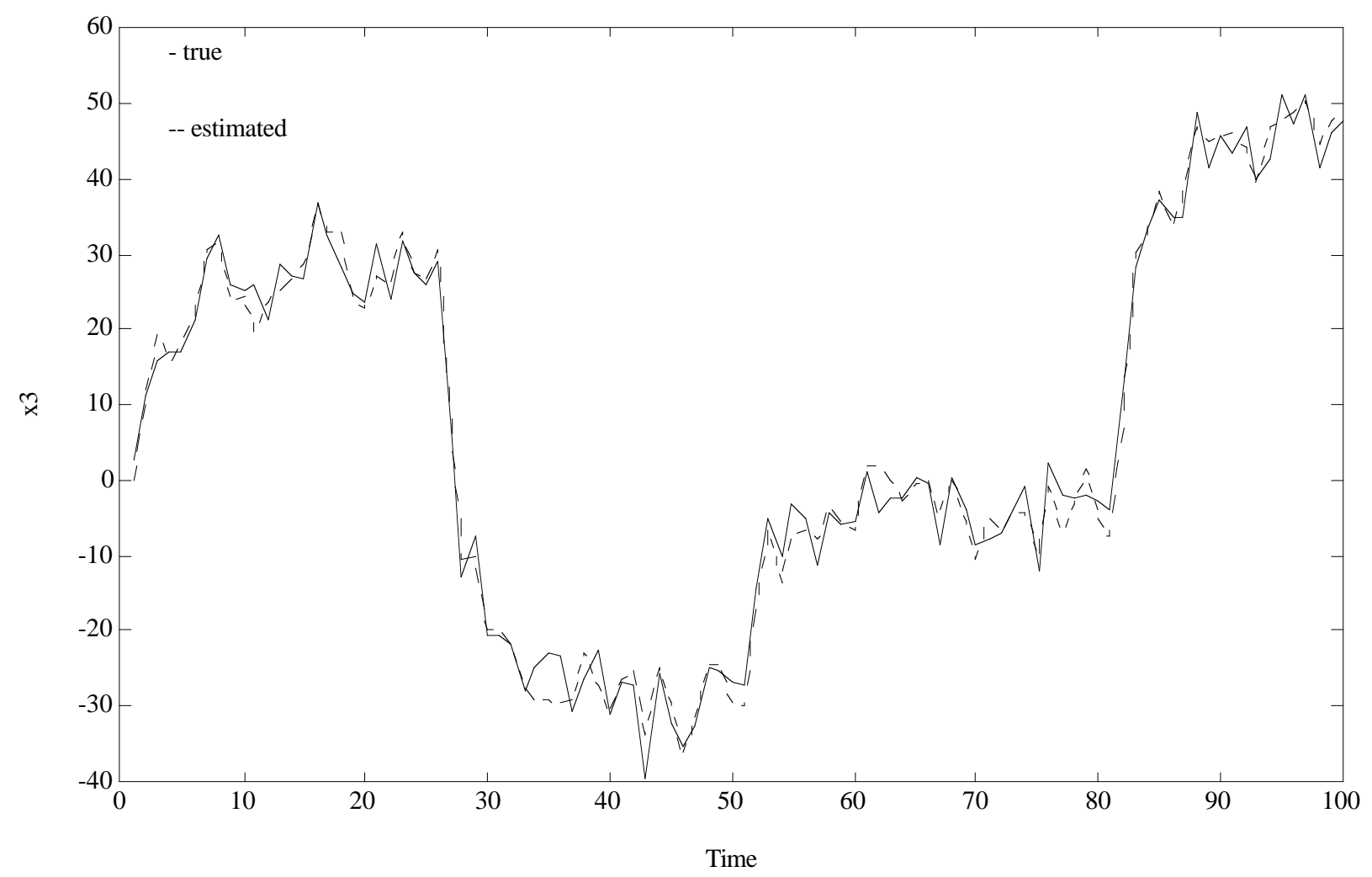

Figure 3. True and estimated state $x_{3}$ 


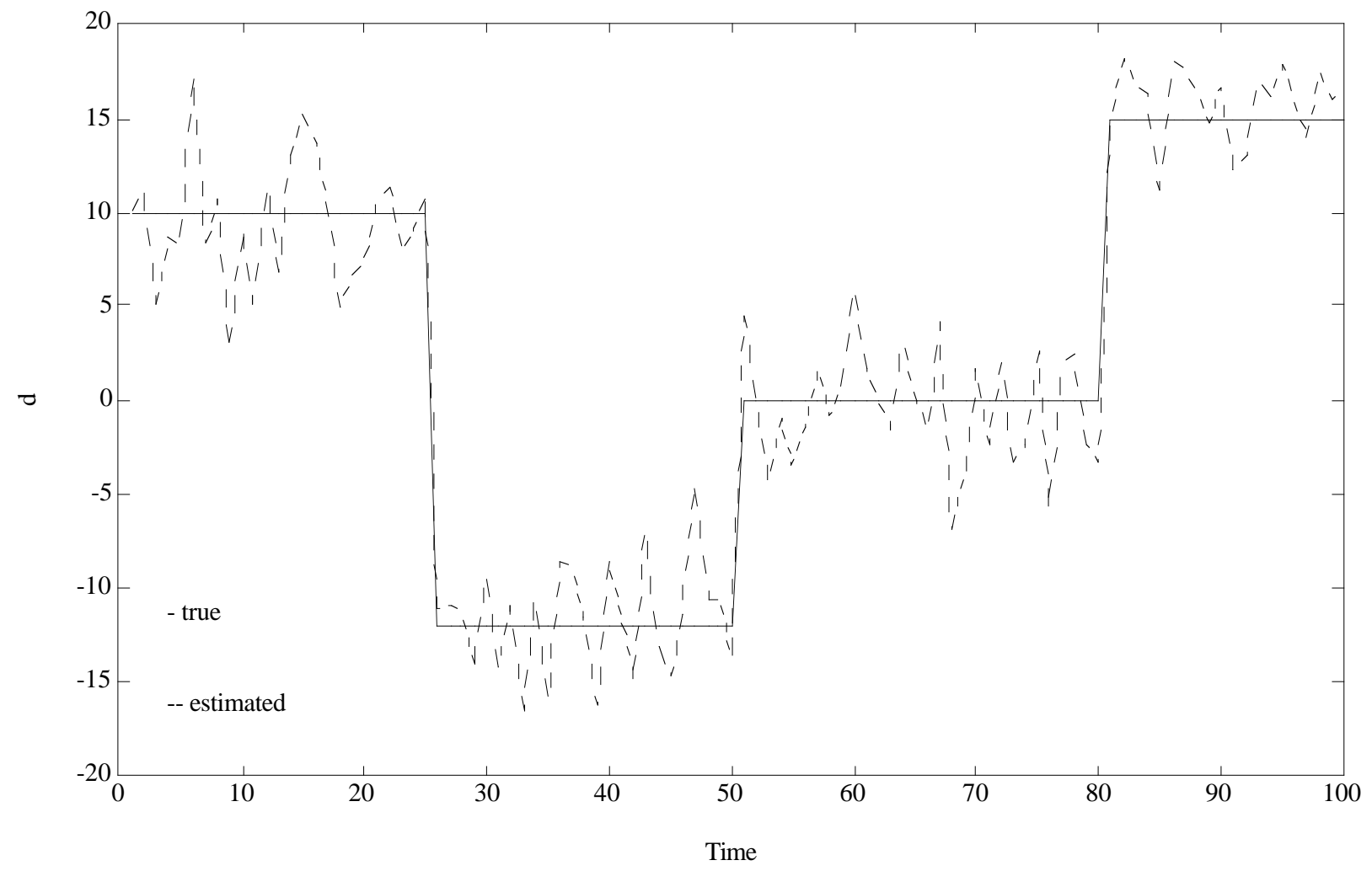

Figure 4. True and estimated unknown input $d$

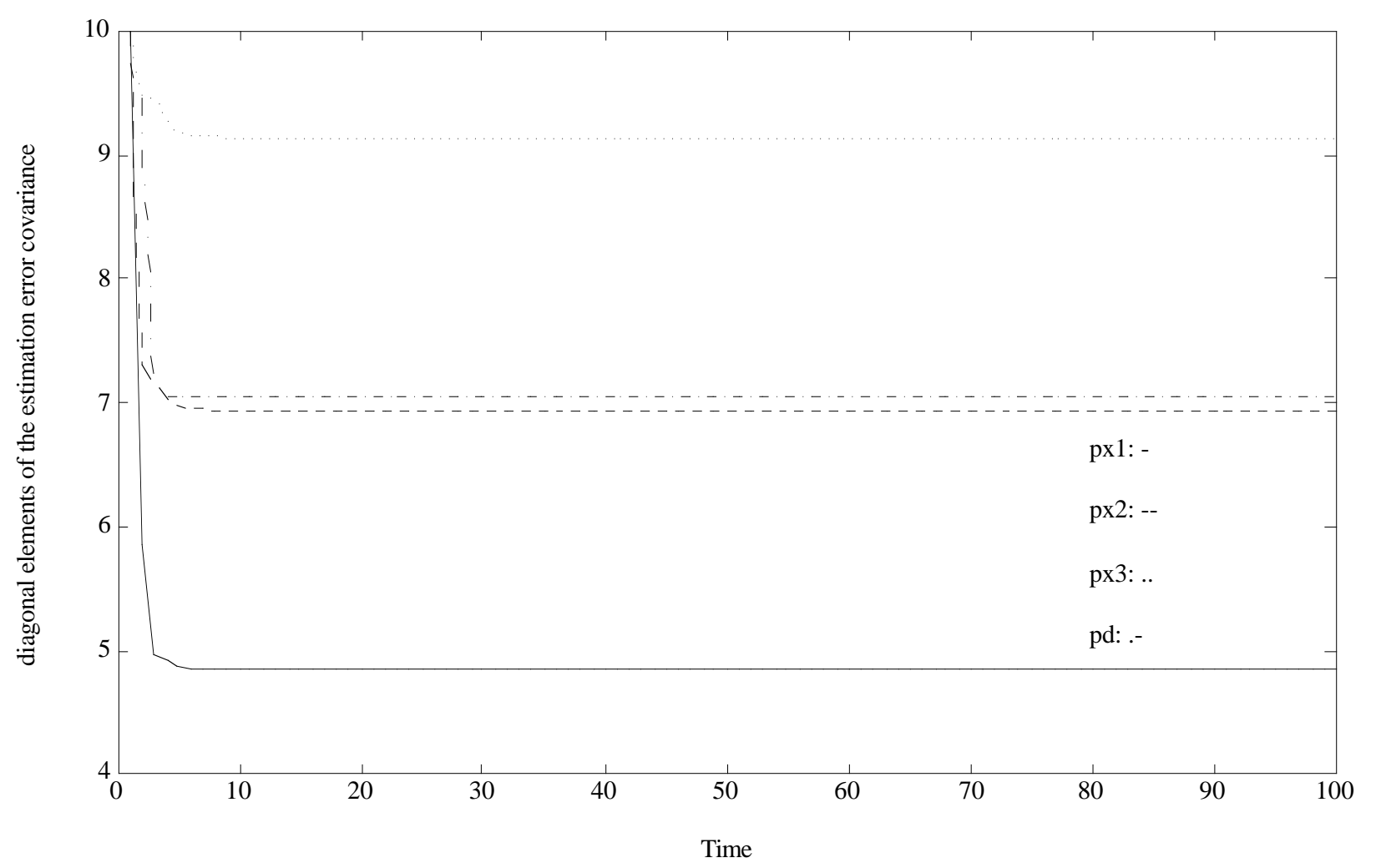

Figure 5. Diagonal elements of the estimation error covariance matrix 


\section{Conclusions}

In this paper, we have developed a new algorithm to estimate the state and unknown inputs for a standard discrete-time system with no assumption on the unknown inputs. By using a singular system's formulation of the system with unknown inputs, we have derived a Kalman filter with unknown inputs. The conditions for existence, convergence and stability of the obtained estimator have been derived. It has been shown that these conditions are not restrictive than those obtained in the previous works on the unknown inputs deterministic observer problem (Hou and Müller 1992).

\section{REFERENCES}

ANDERSON, B.D.O., and MOORE, J.B., 1979, Optimal filtering (Englewood Cliffs, NJ: Prentice Hall).

CAINES, P.E., 1988, Linear stochastic systems (New-York : J. Wiley).

CHANG, C.B., and TABACZYNSKI, J.A., 1984, Application of state estimation to target tracking. IEEE Transactions on Automatic Control, 29, No 2, 98-109.

DAI, L., 1989, Singular control systems. Lecture Notes in Control and Information Sciences, 118 (New-York : Springer Verlag).

DAROUACH, M., ZASADZINSKI, M., and MEHDI, D., 1993, State estimation of stochastic singular linear systems. International Journal of Systems Science, 24, No 2, 345-354.

DAVIS, M.H.A., and VINTER, R.B., 1985, Stochastic Modelling and Control (New-York : Chapman and Hall).

DE SOUZA, C.E., GEVERS, M.R., and GOODWIN, G.C., Riccati equations in optimal filtering of nonstabilizable systems having singular state transition matrices. IEEE Transactions on Automatic control, 31, No 9, 831-838.

GHOLSON, N.H., and MOOSE, R.L., 1977, Manoeuvering target using adaptive state estimation. IEEE Transactions on Aerospace and Electronic Systems, 13, No 3, 310-317.

GUAN, Y., and SAIF, M., 1991, A novel approach to the design of unknown inpout observer. IEEE Transactions on Automatic Control, 36, No 5, 632-635.

HAUTUS, M.L.J., 1969, Controllability and observability conditions of linear autonomous systems. Ned. Akad. Wetenschappen Proceedings, Serie A, 72, 443-448.

HOU, M., and MÜLLER, P.C., 1992, Design of observers for linear systems with unknown inputs. IEEE Transactions on Autmatic Control, 37, No 6, 871-875.

KIRLIN, R.L., and MOGHADDAMJOO, A., 1986, Robust adaptive Kalman filtering for systems with unknown step input and non-gaussian measurement errors. IEEE Transactions on Acoustic, Speech and Signal Processing, 3, No 2, 252-263

MOGHADDAMJOO, A., and KIRLIN, R.L., 1989, Robust adaptive Kalman filtering with unknown inputs. IEEE Transactions on Acoustic, Speech, and Signal Processing, 37, No 8, 1166-1175.

MYERS, K.A., and TAPLEY, B.D., 1976, Adaptive sequential estimation with unknown noise statistics. IEEE Transactions on Automatic Control, 21, No 4, 520-523. 
NIKOUKHAH, R, WILLSKY, A.S., and LEVY, B.C., 1992, Kalman filtering and Riccati equations for descriptor systems. IEEE Transactions on Automatic Control, 37, No 9, 1325-1341.

SINGER, R.A., 1970, Estimating optimal tracking filter performance for manned manoeuvering targets. IEEE Transactions on Aerospace and Electronic Systems, 6, No 4, 473-483.

WANG, S.H., DAVIDSON, E.J., and DORATO, P., 1975, Observing the states of systems with unmeasurable disturbances. IEEE Transactions on Autmatic Control, 20, No 5, 716-717.

YANG F., and WILDE, R.W., 1988, Observers for linear systems with unknown inputs. IEEE Transactions on Automatic Control, 33, No 7, 677-681.

ZASADZINSKI, M., MEHDI, D., and DAROUACH, M., 1991, Recursive state estimation for singular systems. Proceedings of the 1991 American Control Conference, Boston, U.S.A. 